\title{
Cisplatin-resistant osteosarcoma cells possess cancer stem cell properties in a mouse model
}

\author{
JIAN YANG ${ }^{1}$, WEICHUN GUO ${ }^{1}$, LU WANG $^{2}$, LING YU $^{1}$, HONGJUN MEI $^{3}$, \\ SHUO FANG ${ }^{1}$, PENG JI ${ }^{1}$, YANG LIU ${ }^{1}$, GAIWEI LIU ${ }^{1}$ and QI SONG ${ }^{1}$ \\ Departments of ${ }^{1}$ Orthopedics and ${ }^{2}$ Gynecology, Renmin Hospital of Wuhan University, Wuhan, Hubei 430060; \\ ${ }^{3}$ Department of Orthopedics, Wuhan No. 5 Hospital, Wuhan, Hubei 430050, P.R. China
}

Received April 8, 2015; Accepted July 5, 2016

DOI: $10.3892 / \mathrm{ol} .2016 .4956$

\begin{abstract}
Osteosarcoma is the most common malignancy of the bones, and although advances in chemotherapy and surgery had been achieved in recent years, the long-term survival rate has reached a plateau. The main reason for this is the aggressive malignant potential and poor response of the disease to chemotherapy. However, several studies have found that tumor resistance is associated with cancer stem cells (CSCs). To address this issue, in the present study, osteosarcoma cells were treated with specially designated concentrations of cisplatin (CDDP) in a mouse model. Hematoxylin and eosin staining analyses were performed to assess tissue structure, in vivo passaging and CDDP treatment. Drug resistance genes and well-established stemness genes were detected by quantitative polymerase chain reaction. A serum-starved sphere formation assay was adopted to evaluate the ability to generate spherical clones and flow cytometry as used to test the expression of the cluster of differentiation 117 and Stro-1 surface markers, known as markers of CSCs. It was found that CDDP could induce an effect of resistance in the osteosarcoma cells, which possessed cancer stem CSC properties, as shown by the elevated expression of CSC marker genes and the higher expression of the cluster of differentiation 117 and Stro-1 surface markers. Moreover, the cells that dissociated from the tumor tissues exhibited an increased ability to form sarcospheres. The results of this study provided a significant correlation between resistance and CSCs, and revealed a clue indicating that osteosarcoma recurrence is likely to be associated with CSCs.
\end{abstract}

\section{Introduction}

Osteosarcoma is the most frequent primary malignancy of bone tissue, which mostly occurs in adolescents and children,

Correspondence to: Professor Weichun Guo, Department of Orthopedics, Renmin Hospital of Wuhan University, 238 Jiefang Road, Wuhan, Hubei 430060, P.R. China

E-mail: guoweichun@aliyun.com

Key words: osteosarcoma, cisplatin, cancer stem cells, resistant comprising almost $60 \%$ of all bone sarcomas $(1,2)$. Osteosarcoma exists as a local invasion of the bone and soft tissue, and as distant metastases, which most often metastasize to the lung (90\%) and bone (8-10\%), and are rarely observed in the lymph nodes (3). Aggressive removal of the primary tumor via limb sparing surgery or amputation is typically required to ensure local control. Systemic chemotherapy (prior to and following tumor removal) functions in the suppression of metastasis and in the curative intent. The most common chemotherapy regimens are comprised of drugs such as cisplatin (CDDP), doxorubicin and high-dose methotrexate in combination $(4,5)$. Despite neoadjuvant chemotherapy with a wide surgical resection of the tumor, the survival rate for high-grade osteosarcomas remains at only $50-80 \%$ (6) due to its early metastasis and aggressive malignant potential. Nevertheless, a poor response to chemotherapy is usually associated with a poor prognosis (7), and increasing evidence is showing that this therapeutic resistance is partially due to the inherent resistance of a tumorigenic subpopulation of cancer cells, referred to as cancer stem cells (CSCs).

CSCs were firstly isolated in myeloid leukemia (8), and recent studies have indicated that osteosarcoma is also driven by this small subpopulation of cells (9), which possess the potential for self-renewal and the ability to differentiate and proliferate. Although the drug resistance mechanisms of CSCs remain unclear, several studies have highlighted that the adenosine triphosphate-binding cassette drug transporters and chemotherapy-metabolizing enzyme expression may be involved, as well as changes in cell cycle kinetics and anti-apoptotic protein overexpression (10-12). Unlike in epithelial cancer, the specific phenotypes of CSCs in osteosarcoma have not yet been defined. As revealed by Adhikari et al (13), CD117 and Stro-1 are potential candidates for osteosarcoma CSC markers that are associated with metastasis and drug resistance, the most lethal characteristics of the disease. To the best of our knowledge, the association between tumor drug resistance in osteosarcoma cells and CSCs has rarely been discussed; therefore, the present study used CDDP, which is frequently applied for sarcoma treatment, to demonstrate the resistance of osteosarcoma cells possessing CSC properties in a mouse model.

In the present study, the effects of CDDP on CSCs were assessed using the osteosarcoma 143B-TK cell line in in vivo 
conditions. The results demonstrated that CDDP induced the resistance of osteosarcoma cells possessing CSC properties in vivo.

\section{Materials and methods}

Cell culture. The human osteosarcoma 143B-TK cell line was obtained from the China Center for Type Culture Collection (Wuhan, China) and cultured in Hyclone Dulbecco's minimum essential medium (DMEM; GE Healthcare Life Sciences, Logan, UT, USA) with $10 \%$ fetal bovine serum FBS and $1 \%$ penicillin/streptomycin (Thermo Fisher Scientific Inc., Waltham, MA, USA). The cells were kept at $37^{\circ} \mathrm{C}$ in a $5 \% \mathrm{CO}_{2}$ humidified atmosphere, and harvested just as they reached $\sim 70 \%$ confluence.

In vivo animal model. Female, non-obese diabetic/severe combined immunodeficiency (NOD/SCID) mice (3-5 weeks old) were obtained from the Animal Biosafety Level-3 Laboratory of Wuhan University (Wuhan, China) and housed in individually vented cages under specific pathogen-free conditions, with a $12 \mathrm{~h}$ day/night cycle, and food and water ad libitum. The osteosarcoma 143B-TK cell line was cultured as aforementioned, and $\sim 10^{6}$ of the cells were resuspended in $100 \mu 1$ phosphate-buffered saline (PBS), which was injected subcutaneously into the neck of each mouse. The animals were evaluated for the incidence of tumor formation and checked weekly for tumor progression; each measurement consisted of two diameters, length (a) and width (b), and the size of tumors were calculated by using the following formula: Volume $=0.2$ $618 \times$ a $\times$ b x $(a+b)(14)$. The mice that grew a tumor mass were randomly divided into two groups when the tumors reached to $\sim 50-\mathrm{mm}^{3}$. One group served as the CDDP-resistant group and received an intraperitoneal injection of CDDP (Thermo Fisher Scientific Inc.) at a dose of $5 \mathrm{mg} / \mathrm{kg} /$ week over a period of 4 weeks. The other group served as the control group and was administered $100 \mu 1 \mathrm{PBS}$ only by intraperitoneal injection. The mice were monitored for up to 90 days, after which they were humanely euthanized with $\mathrm{CO}_{2}$. All experiments were performed according to protocols approved by the Institutional Animal Care and Use Committee and the Institutional Biosafety Committee of the Animal Biosafety Level-3 Laboratory of Wuhan University.

Hematoxylin and eosin (HE) staining of the tumors. Tumors were obtained from the mice as aforementioned, and then fixed with $4 \%$ paraformaldehyde overnight and embedded in paraffin. Paraffin-embedded tissues sections ( $4-\mu \mathrm{m}$ thick) were deparaffinized and stained with hematoxylin (Sigma-Aldrich, St. Louis, MO, USA) for $8 \mathrm{~min}$. Subsequent to being washed with PBS and Aqua Dest, the sections were incubated with eosin (1:100 in Aqua Dest; Sigma, St. Louis, USA) for $3 \mathrm{~min}$. Afterwards, the sections were washed with Aqua Dest, embedded in the mounting medium, covered with a coverslip and analyzed by transmission light microscopy (Olympus BX51; Olympus, Tokyo, Japan).

Reverse transcription-polymerase chain reaction (RT-qPCR). Total RNA was isolated and dissolved in $20 \mu \mathrm{l}$ RNA enzyme-free water and then $2 \mu \mathrm{l}$ of sample was reverse transcribed per assay. qPCR was then performed using an ABI 7900 System (Applied Biosystems; Thermo Fisher Scientific Inc.) in the presence of SYBR Green. The following gene-specific primers were used: Multi-drug resistance association protein-1 (MRP-1) forward, 5'-CTGGCTTGGTGT GAACTGAT-3' and reverse, 5'-AGGCTCTGGCTTGGCTCT AT-3'; multi-drug resistance gene-1 (MDR-1) forward, 5'-GGA CAGAAACAGAGGATCGC-3' and reverse, 5'-CCCGTCTTG ATCATGTGGCC-3'; octamer-binding transcription factor 4 (OCT4) forward, 5'-GAGTGAGAGGCAACCTGGAGAAT-3' and reverse, 5'-ACCGAGGAGTACAGTGCAGTGAA-3'; SRY (sex determining region Y)-box 2 (SOX2) forward, 5'-TGG GTTCGGTGGTCA AGTCC-3' and reverse, 5'-TGTGTG AGAGGGGCAGTGTG-3'; telomerase reverse transcriptase (TERT) forward, 5'-AAGTTTGGAAGAACCCCACAT T-3' and reverse, 5'-AGGATGGTCTTGAAGTCTGAGG-3'; Nanog forward, 5'-GAGAAGAGTGTCGCAAAAAAGGA-3' and reverse, 5'-TGAGGTTCAGGATGTTGGAGAGT-3'; and $\beta$-actin forward, 5'-CACCCAGCACAATGAAGATCAAGA T-3' and reverse, 5'-CCAGTTTTTAAATCCTGAGTCAAG C-3'. The expression of the TERT, Nanog, OCT4 and SOX2 marker genes is associated with CSCs, and $\beta$-actin was used as endogenous normalization control. Target sequences were amplified at $95^{\circ} \mathrm{C}$ for $10 \mathrm{~min}$, followed by 40 cycles of $95^{\circ} \mathrm{C}$ for $15 \mathrm{sec}$ and $60^{\circ} \mathrm{C}$ for $1 \mathrm{~min}$. All assays were performed in triplicate. The fold-change in mRNA expression was determined according to the $2^{\Delta \Delta C q}$ method (15).

Isolation of tumor cells. After the mice were sacrificed at the endpoint of therapy, the tumors in each group were collected and immediately immerged in DMEM supplemented with $10 \%$ FBS and $1 \%$ penicillin/streptomycin. The tumors were transported in a container filled with liquid nitrogen, and then the necrotic tissue of the tumor was removed and the tumors were cut into small sections under sterile conditions. Trypsin solution $(0.25 \%)$ with ethylenediaminetetraacetic acid (EDTA; $0.02 \%$ ), at 10 times of the volume of the tumor tissue, was added for digestion for $40 \mathrm{~min}$ at $37^{\circ} \mathrm{C}$ in a shaking incubator, and then the digestive fluid was discarded and the cells were resuspended in $1 \mathrm{ml}$ DMEM to obtain a homogenized cell suspension. The suspension was diluted in DMEM with $10 \%$ FBS and $1 \%$ penicillin/streptomycin and incubated under standard conditions $\left(37^{\circ} \mathrm{C}\right.$ and $\left.5 \% \mathrm{CO}_{2}\right)$ in a humidified atmosphere. Just as the cells began to attach to the bottom of the plate, the medium was replaced every second day.

Sphere formation assay. At $70 \%$ confluence, the tumor cells in DMEM supplemented with $10 \%$ FBS medium were dissociated with trypsin-EDTA into single-cell suspensions. A total of $1 \times 10^{5}$ cells/well were seeded in ultra-low attachment 6-well plates (Corning Inc., Corning, NY, USA) and inoculated into RPMI-1640 medium without serum, supplemented with $2 \%$ B27 solution, human epidermal growth factor (EGF; $20 \mathrm{ng} / \mathrm{ml}$ ) and human basic fibroblast growth factor (b-FGF; $20 \mathrm{ng} / \mathrm{ml}$ ). After 2 weeks, the resultant spheroids that contained $>20$ cells were counted under an inverted phase contrast microscope (Olympus IX51). Subsequently, the spheres were dissociated and re-introduced into another 6-well ultralow attachment plate to investigate their ability of self-renewal through a secondary sphere formation assay. 
Flow cytometry analysis. Isolated cells were cultured in DMEM and dissociated with trypsin-EDTA into single-cell suspensions as aforementioned. Subsequent to being washed with PBS twice, the cells were harvested and incubated with cluster of differentiation (CD)117-phycoerthyrin (dilution, 1:20; catalog no., 313203) and Stro-1 (dilution, 1:20; catalog no., 340103) mouse monoclonal antibodies [in PBS $(\mathrm{pH} 7.2)$ containing $0.09 \%$ sodium azide and $0.2 \%(\mathrm{w} / \mathrm{v})$ bovine serum albumin (Sigma-Aldrich); BioLegend, Inc., San Diego, CA, USA] on ice for $30 \mathrm{~min}$ in the dark. Afterwards, the cells were washed twice with PBS and kept at $4^{\circ} \mathrm{C}$ in the dark prior to flow cytometry (BD FACSCalibur; BD Biosciences Franklin Lakes, NJ, USA) and analyzed using BD Cell Quest Pro software version 5.1 (BD Biosciences). The expression of the cell markers was determined by comparison with an isotype control.

Statistical analysis. Statistical analyses were performed using the SPSS 13.0 statistical software package (SPSS Inc., Chicago, IL, USA). Data are expressed as the mean \pm standard deviation of three independent experiments. The Student's $t$-test was used to compare the means of the 2 groups. When $>3$ means were compared, one-way analysis of variance, followed by multiple comparisons among the means, was used. $\mathrm{P}<0.05$ was considered to indicate a statistically significant difference.

\section{Results}

Metronomic CDDP therapy significantly delays the growth of tumors in vivo. Female NOD/SCID mice were subcutaneously injected with human osteosarcoma 143B-TK cells and only 12 mice (12/18) finally formed a tumor xenograft. Half of these mice were treated with metronomically scheduled CDDP ( $5 \mathrm{mg} / \mathrm{kg}$ every week applied intraperitoneally), and drug treatment was started at the third week after tumor cell implantation, just when tumors reached an average volume of $50 \mathrm{~mm}^{3}$. Regularly CDDP treatment resulted in a significant decrease in the size of the tumor and delayed the tumor growth (Fig. 1). The tumor volume of the treated mice was slowly reduced to $60 \mathrm{~mm}^{3}$ at the eighth week after tumor cell implantation, whereas the tumors in the control group exhibited rapid growth, with at a growth rate of $40 \%$. At around the ninth week after tumor cell implantation, the tumor volume began to increase in the CDDP treatment group despite ongoing treatment, with a tumor growth rate of $85 \%$, while tumors in the control group were constant in volume at $\sim 6,000 \mathrm{~mm}^{3}$, with a sharp slowing of tumor growth (Fig. 1A). Treatment was stopped at the seventh week and the two groups of mice were sacrificed in the twelfth week; at this endpoint, tumors were collected and subjected to histological and macroscopic analyses. Meanwhile, the tumor cells were isolated from the tumor tissue for characterization and cell experiments.

Effect of CDDP therapy on tumor macroscopic appearance. Tumor tissues from the two groups were macroscopically assessed at the therapy endpoint. The tumor tissue in the CDDP treatment group appeared pale and dingy, was harder and had less blood supply on the surface of the tissues compared with the untreated control tumors (Fig. 1B and C). For further evaluation of the changes induced by in vivo passaging and
CDDP treatment, $\mathrm{HE}$ staining analyses were performed in the treatment and control groups; sections were stained with $\mathrm{HE}$ and analyzed by transmitted light microscopy. The tissue structure in the original 143B-TK xenografts (established from the control group) was compact and homogeneous, with a large number of tumor cells that were arranged irregularly and no hybrid connective tissue between the tumor cells (Fig. 1E). The resistant tumors (treatment group) exhibited an inhomogeneous structure and the number of osteosarcoma cells was significantly reduced, with hyperplasia of the connective tissue (Fig. 1D). This indicated that CDDP exhibited a strong toxic effect on the tumor cells; resistance tissues underwent a series of corresponding changes in structure compared with the control tissues in the mouse model.

Expression profiles of drug resistance and stemness genes in response to $C D D P$ therapy in vivo. For characterization of stemness as a possible cause of tumor cell drug resistance and recurrence, the drug resistance genes, MDR1 and MRP1, and the well-established stemness genes, OCT4, SOX2, TERT and Nanog, were detected by qPCR after samples were extracted from the tumor tissue. The analysis results revealed that the expression levels of MRP1 (Fig. 2A), MDR1 (Fig. 2B), OCT4 (Fig. 2C), SOX2 (Fig. 2D), TERT (Fig. 2E) and Nanog (Fig. 2F) were significantly elevated from 0.15 - to 10 -fold in resistant tumors tissues in comparison to tumors established from untreated mice, which indicated that the proportion of stem cells was increased in osteosarcoma tissues from CDDP-treated mice. In the CDDP group, MRP-1 expression showed a significant increase $(\mathrm{P}=0.026), \mathrm{MDR}-1$ expression increased slightly $(\mathrm{P}=0.041)$ and the expressions of the stem cell-related genes OCT-4, SOX-2, TERT and Nanog were elevated significantly $(\mathrm{P}=0.021,0.029,0.043$ and 0.031 , respectively) compared with the control group. The particular mechanism of this phenomenon remains unclear, and we speculated that there were a number of CSCs in the osteosarcoma tissues that had a stronger tolerance to CDDP. In other words, CDDP could select and enrich the CSCs in osteosarcoma tissues in vivo.

Cells resistant to CDDP show higher expression of CSC markers CD117 and Stro-1. To confirm the percentage of putative osteosarcoma stem cells, cells cultured from the tumor tissues of the two groups were tested by flow cytometry for the expression of surface markers CD117 and Stro-1, known markers of CSCs. The percentage of CD117 $7^{+}$Stro- $1^{+}$ cells in the resistant group was $0.5 \%$ (Fig. 3A), where $<0.1 \%$ of $\mathrm{CD} 117^{+}$Stro- $1^{+}$cells were detected in the control group (Fig. 3B). Despite the percentage of double-positive markers being low in the resistant group, a distinct difference was found in the CDDP untreated cells, which provided direct evidence for CDDP-resistant osteosarcoma cells that possess CSC properties.

CDDP-resistant cells exhibit an increased ability to form sarcospheres. For characterization of cell dependency on essential matrix signaling, the ability to generate spherical clones and the ability for self-renewal were evaluated in a serum-starved sphere formation assay. Within several days after being cultured in RPMI-1640 medium without serum, 

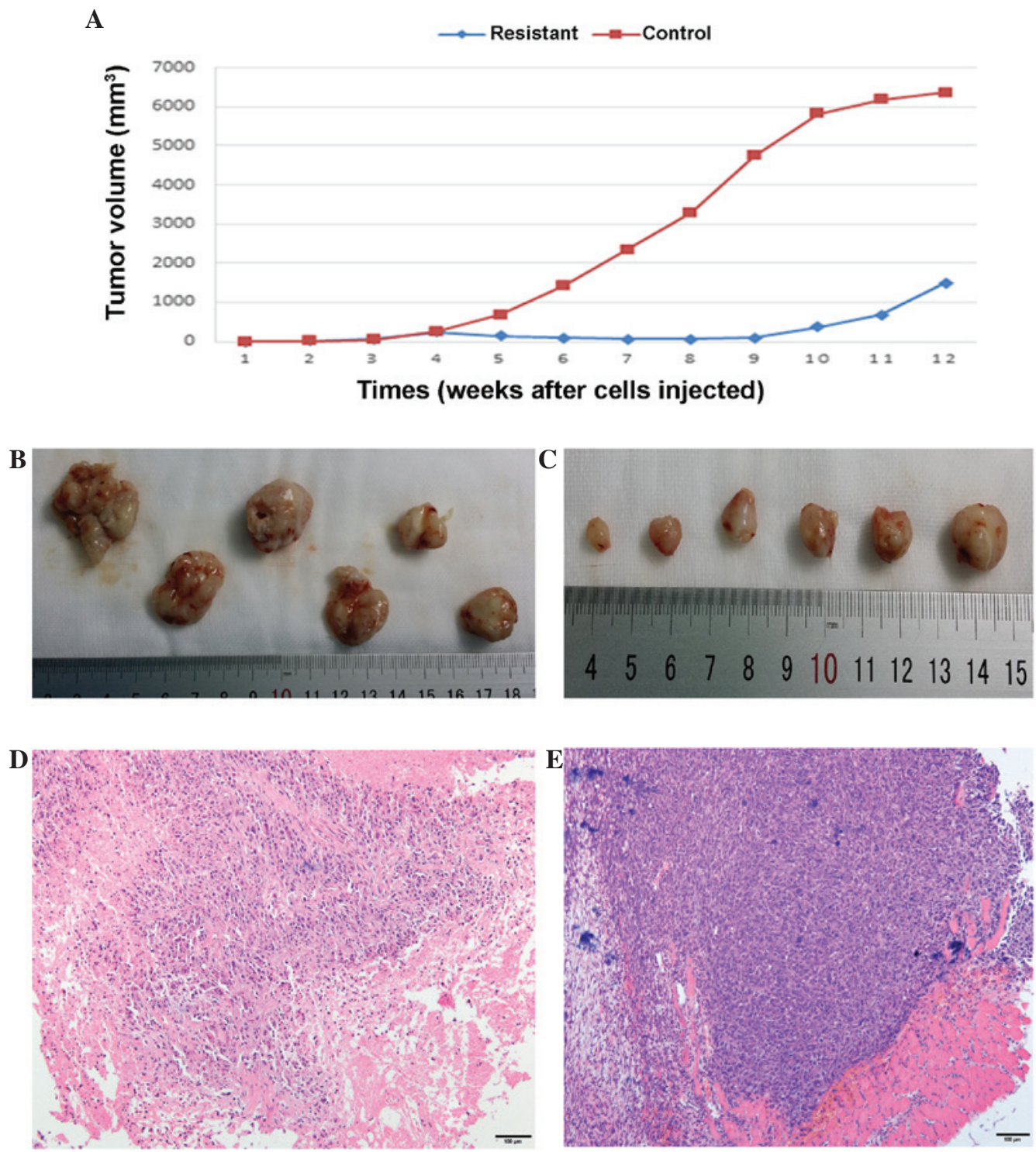

Figure 1. Effect of cisplatin (CDDP) on osteosarcoma tissue growth and macroscopic appearance in the mouse model. Tumor xenografts were formed by osteosarcoma 143B-TK cell injection into two groups; (A) CDDP treatment was started on the third week and lasted for 4 weeks in the resistant group, which exhibited a significantly delay in the growth of the tumor compared with the control group. Tumor tissues from the (B) resistant and (C) control groups were macroscopically assessed at the therapy endpoint, and hematoxylin and eosin staining analyses were performed in the (D) resistant and (E) control groups, which showed marked differences (magnification, x100).

the cells started to form spherical colonies, and significant differences between control and resistant group cells could be observed after 2 weeks (Fig. 3C and D), as the spheroids in the control group appeared smaller in size and fewer in number, and the spheroids in resistant group were more compact and homogeneous. The spheroids eventually formed at a frequency of $\sim 1 / 42\left(2,373.33 \pm 133.2\right.$ colonies $/ 1 \times 10^{5}$ cells $)$ for the resistant group, and $1 / 113\left(887 \pm 14.45\right.$ colonies $/ 1 \times 10^{5}$ cells) for the control group (Fig. 3E). To investigate cell self-renewal potential in vitro, cultured spheres were dissociated into single cells and allowed to grow in serum-starved medium supplemented with B27, human EGF and human b-FGF repeatedly. Sarcospheres from each group showed expansion in the suspension culture, which led to cell differentiation and self-renewal through the formation of secondary spheres. The frequency of sphere formation was similar to that of the primary sphere results, with $\sim 1 / 117\left(85.5 \pm 2.15\right.$ colonies $/ 1 \times 10^{4}$ cells) for the resistant group compared with $1 / 388\left(25.75 \pm 1.35\right.$ colonies/ $1 \times 10^{4}$ cells $)$ for the control group (Fig. 3F). The number of generate spheroids and secondary spheres in CDDP-resistant cells was increased significantly ( $\mathrm{P}=0.039$ and 0.045 , respectively) compared with the control group.

\section{Discussion}

Osteosarcoma is a highly malignant form of bone cancer with the characteristic of osteoid produced in tumor tissues $(1,2,16)$. Despite numerous improvements in the therapeutic strategies for osteosarcoma, problems remain during the course of treatment, such as therapeutic resistance, cancer recurrence and metastatic disease, and usually tumor drug resistance is closely associated with recurrence. Currently, there are a large number of studies regarding the development and use of xenografts and allograft models of human and osteosarcoma cells 
A

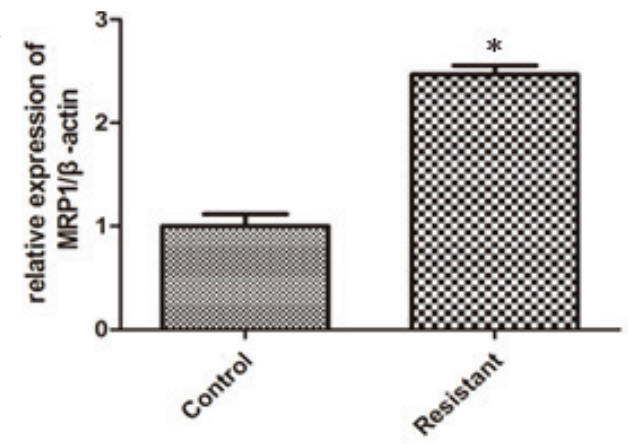

C

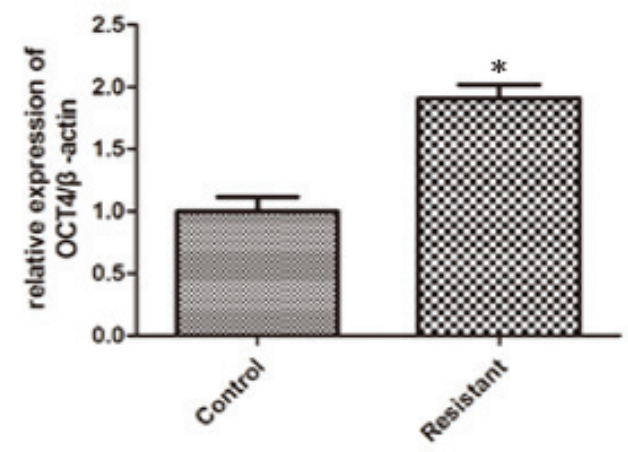

E

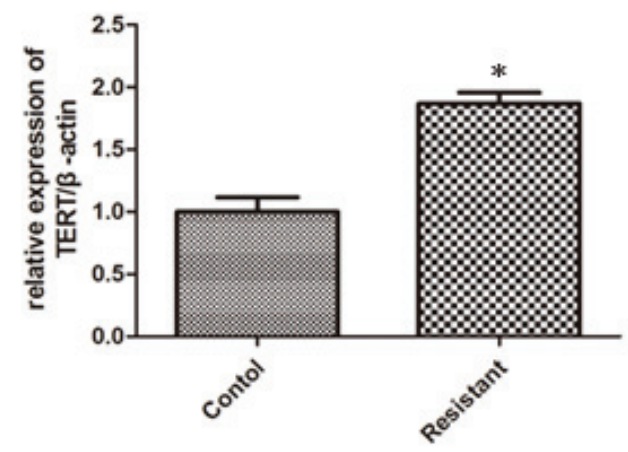

B

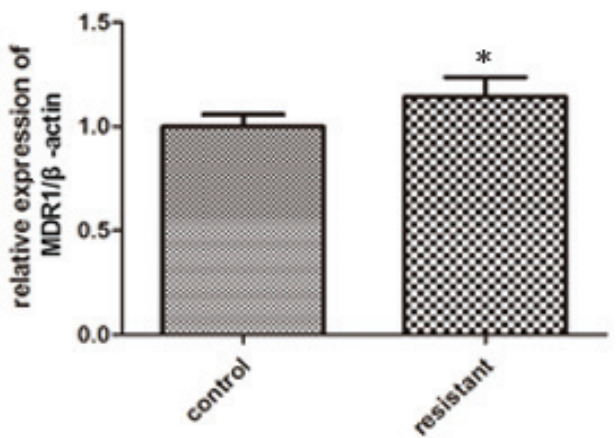

D

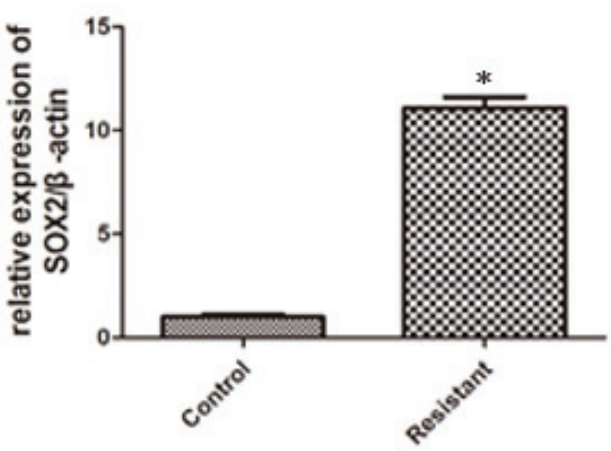

F

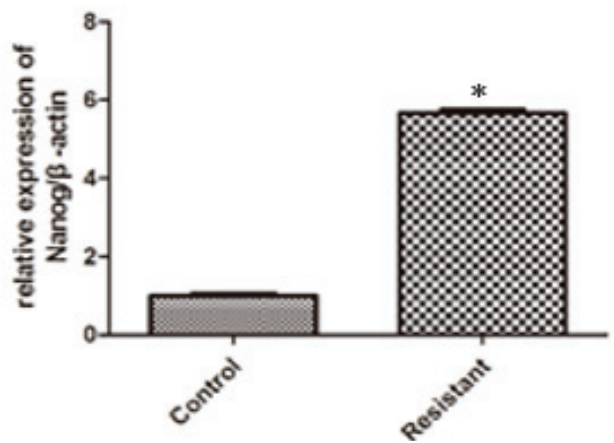

Figure 2. Drug resistance genes and stemness genes were activated by cisplatin (CDDP) in vivo. The osteosarcoma tissues were cultured with or without CDDP in the mouse model. (A and B) Quantitative polymerase chain reaction (qPCR) data showed that the treatment of the osteosarcoma tissues with CDDP resulted in the increased expression of the drug resistance genes, (A) multi-drug resistance association protein-1 (MRP1) and (B) multi-drug resistance gene-1 (MDR1), (C-F) qPCR data showed that the expression of the stemness genes, octamer-binding transcription factor 4 (OCT4), SRY (sex determining region Y)-box 2 (SOX2), telomerase reverse transcriptase (TERT) and Nanog, was elevated following treatment with CDDP. Results represent the mean \pm standard deviation of three individual experiments. " $\mathrm{P}<0.05$ vs. control.

injected into immunocompromised mice. These models have yielded tumors histologically resembling human cancer and have produced cell lines to complement human osteosarcoma studies (17). The advantages of a model using the injection of a subcutaneous cell suspension are its high incidence rate and good reproducibility $(18,19)$. A multitude of studies of chemotherapy resistance are based on cells, and osteosarcoma models are rarely used for evaluating the histological and molecular characteristics of drug resistance. In the present study, CDDP was used to create an animal model of drug resistance, and it was found that CDDP can significantly reduce the tumor volume and result in a significant delay in tumor growth. Moreover, the products of the multi-drug resistance genes MDR1 and MRP1, which act as an energy-dependent elimination pump that can convey cytotoxic drugs out of tumor cells (20), have previously been perceived as prognostic factors in osteosarcoma (21). In the present study, RT-qPCR revealed that the MDR1 and MRP1 genes were overexpressed in the CDDP treatment group compared with in the untreated tissues, indicating that MDR1 and MRP1 expression levels increase under chemotherapeutic treatment, which is similar to the results reported by other studies regarding the treatment of osteosarcoma (22-24). This result also certified that the establishment of an animal resistance model induced by CDDP via the aforementioned method is feasible and effective.

Recent studies have suggested that CSCs are involved in the mechanisms of drug resistance (25-27), providing a novel theoretical basis to solve the problem of chemotherapy resistance during the eradication of osteosarcoma. At present, identifying and isolating CSCs mainly relies on surface markers or side population sorting. However, isolating and purifying CSCs is occasionally difficult due to their continuous differentiation and scarcity. Previous studies have shown that OCT4 expression is vital in the maintenance of stem cell pluripotency (28), and that SOX2 and Nanog expression contributes to plasticity, self-renewal and stemness (29). The present results revealed that OCT4, SOX 2 and Nanog expression was increased in the CDDP treatment group compared with the untreated control 
A

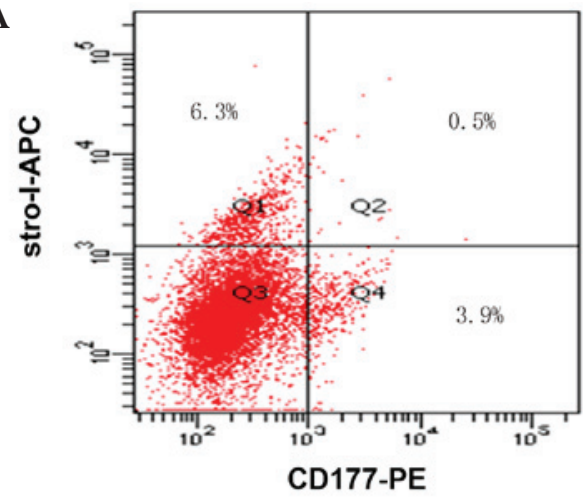

$\mathbf{C}$

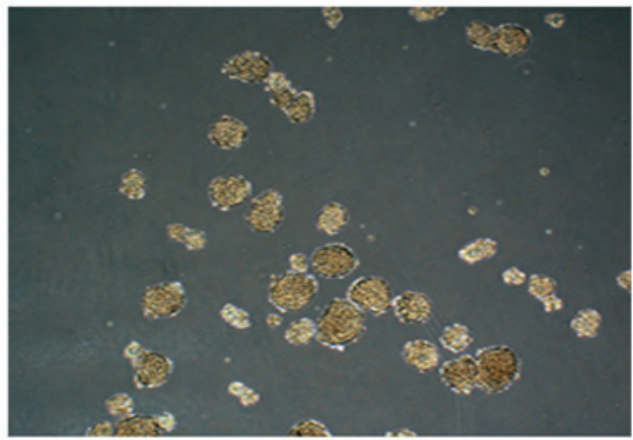

$\mathbf{E}$

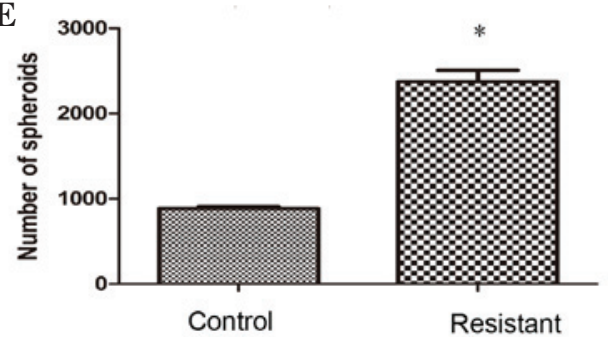

B
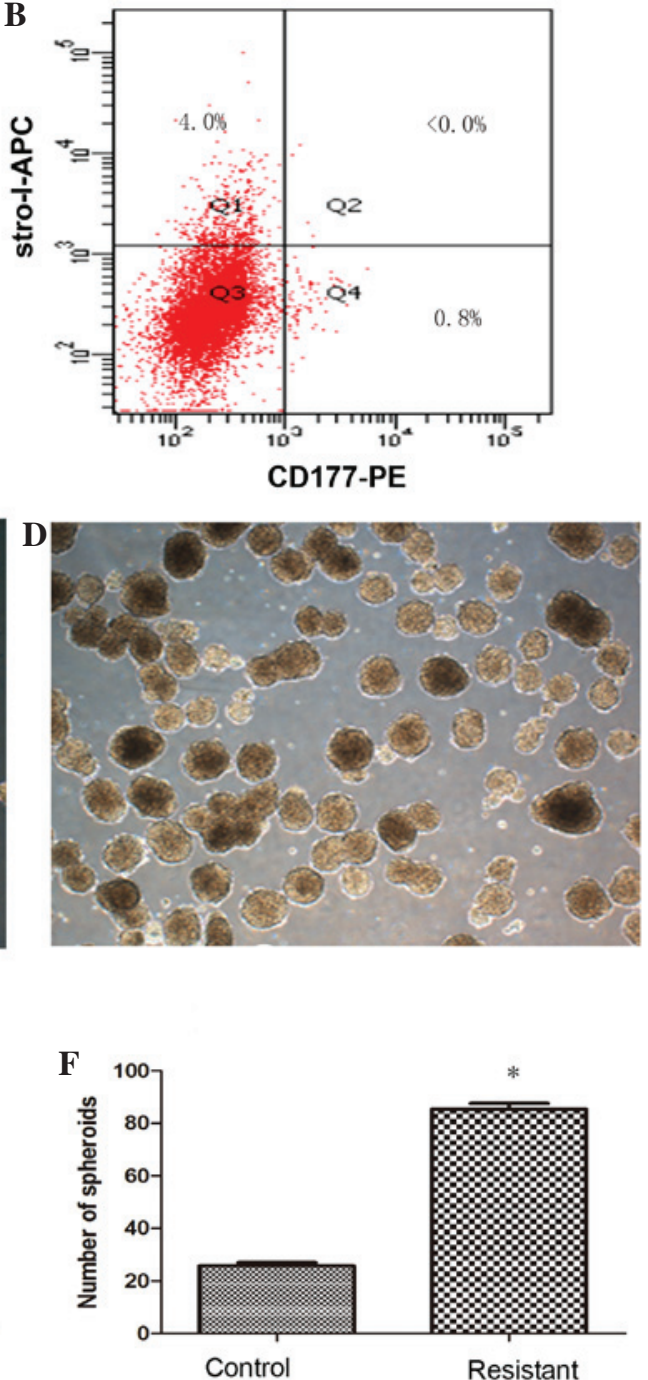

Figure 3. Cisplatin (CDDP)-resistant cells exhibit increased cancer stem cell (CSC) properties. The surface markers of CSCs were tested by flow cytometry in cells cultured from the of tumor tissue, and the percentage of CD117 $7^{+}$Stro- $1^{+}$cells in the (A) resistant group $(0.5 \%)$ showed higher expression of CD117 and Stro-1 compare with the cells of the (B) control group $(<0.0 \%)$, while single positive CD117 or Stro-1, and double-negative CD117-Stro1 ${ }^{-}$cells in the resistant group were exhibited at 3.9,6.3 and 89.3\%, respectively, compared with 0.8, 4.0 and $95.2 \%$ in the control group Differences in size and number appeared in the forming spherical colonies in the (C) control and (D) resistant groups. The ability to generate spheroids in a serum-starved sphere formation assay was increased in the (E) CDDP-resistant cells, and this result was also similar in (F) the formation of secondary spheres. Results represent the mean \pm standard deviation of three repeated experiments. "P<0.05 vs. control. APC, allophycocyanin; PE, phycoerythrin.

group. This indicated that the CDDP-resistant cells in tumor tissues possessed CSC characteristics that increased the expression of the stem cell-related genes and the expression of the surface markers, CD117 and Stro-1, as determined by an increased percentage of $\mathrm{CD} 117^{+}$Stro- $1^{+}$tumor cells isolated from the drug treatment tissues. Although the level of $\mathrm{CD} 117^{+}$Stro- $1^{+}$cells in the tumor tissues, as detected by immunohistochemistry analysis, was unsatisfactory [albeit ascended from $<0.1 \%$ (Fig. 3B) to $5 \%$ (Fig. 3A)], we speculate on the possibility that CD117 and Stro-1 are expressed at low levels in osteosarcoma and that CSCs are rare in the tumor. Similar results were also found in the sphere formation assay, which showed that CDDP-resistant cells exhibited an increased ability to form spheres and secondary spheres compared with the untreated cells, meaning that the cells possessed the potential for self-renewal and that the ability for tumorigenicity were strengthened by CDDP though this method. It is known that CD117 (c-kit) is the receptor for stem cell factor and a proto-oncoprotein, and that Stro- 1 is a cell surface marker for mesenchymal stem cells (30), nonetheless, it remains unknown whether CD117 or Stro-1 plays an active role in the properties of osteosarcoma-initiating cells. Adhikara et al (13) documented that CD117 and Stro-1 were preferentially expressed in drug-resistant cells and spheres. High CD $117^{+}$Stro- $1^{+}$expression and the results of a sphere formation assay in CDDP-treated cells in the present study confirmed that the resistance tumor possessed a strong ability for oncogenicity and self-renewal; in other words, CDDP induced and enriched the CSCs in the xenograft tissues.

In conclusion, in the present study, acquired in vivo chemoresistance against CDDP treatment was studied in a human osteosarcoma 143B-TK xenograft mouse model. The results showed that CDDP could induce the stem cell-related genes and the expression of the surface markers, enhance the characterization of stem cells and enrich the CSCs in the xenograft tissues. This suggests an association between chemoresistance 
and the properties of CSCs, indicating that osteosarcoma cells probably acquired the ability to evade the chemotherapy drug, CDDP, through upregulating the CSC-related gene. In addition, this result also indicates that osteosarcoma recurrence is probably associated with CSCs. Although there was a lack of data on the differentiation and metastasis of the tumor, which requires investigation in further research, CDDP-resistant osteosarcoma cells showed CSC properties in the mouse model. Further study on how the specific mechanisms of resistance may impact cell plasticity and differentiation remain to be investigated in future.

\section{Acknowledgements}

The study was supported by a grant from the Wuhan science and Technology Council of Hubei Province (no. 2014062801011264).

\section{References}

1. Bielack SS, Kempf-Bielack B, Delling G, Exner GU, Flege S, Helmke K, Kotz R, Salzer-Kuntschik M, Werner M, Winkelmann W, et al: Prognostic factors in high-grade osteosarcoma of the extremities or trunk: An analysis of 1,702 patients treated on neoadjuvant cooperative osteosarcoma study group protocols. J Clin Oncol 20: 776-790, 2002.

2. Cormier JN and Pollock RE: Soft tissue sarcomas. CA Cancer J Clin 54: 94-109, 2004.

3. Luetke A, Meyers PA, Lewis I and Juergens H: Osteosarcoma treatment-where do we stand? A state of the art review. Cancer Treat Rev 40: 523-532, 2014.

4. Janeway KA and Grier HE: Sequelae of osteosarcoma medical therapy: A review of rare acute toxicities and late effects. Lancet Oncol 11: 670-678, 2010

5. Picci P, Ferrari S, Bacci G and Gherlinzoni F: Treatment recommendations for osteosarcoma and adult soft tissue sarcomas. Drugs 47: 82-92, 1994.

6. Meyers PA: Muramyl tripeptide (mifamurtide) for the treatment of osteosarcoma. Expert Rev Anticancer Ther 9: 1035-1049, 2009

7. Gorlick R, Anderson P, Andrulis I, Arndt C, Beardsley GP, Bernstein M, Bridge J, Cheung NK, Dome JS, Ebb D, et al: Biology of childhood osteogenic sarcoma and potential targets for therapeutic development: Meeting summary. Clin Cancer Res 9: 5442-5453, 2003

8. Bonnet D and Dick JE: Human acute myeloid leukemia is organized as a hierarchy that originates from a primitive hematopoietic cell. Nat Med 3: 730-737, 1997.

9. Park CY, Tseng D and Weissman IL: Cancer stem cell-directed therapies: Recent data from the laboratory and clinic. Mol Ther 17: 219-230, 2009.

10. Dean M, Fojo T and Bates S: Tumour stem cells and drug resistance. Nat Rev Cancer 5: 275-284, 2005.

11. Korkaya $\mathrm{H}$ and Wicha MS: Selective targeting of cancer stem cells: A new concept in cancer therapeutics. BioDrugs 21: 299-310, 2007.

12. Ma S, Lee TK, Zheng BJ, Chan KW and Guan XY: CD133 HCC cancer stem cells confer chemoresistance by preferential expression of the Akt/PKB survival pathway. Oncogene 27: 1749-1758, 2008.
13. Adhikari AS, Agarwal N, Wood BM, Porretta C, Ruiz B, Pochampally RR and Iwakuma T: CD117 and Stro-1 identify osteosarcoma tumor-initiating cells associated with metastasis and drug resistance. Cancer Res 70: 4602-4612, 2010.

14. Luu HH, Kang Q, Park JK, Si W, Luo Q, Jiang W, Yin H, Montag AG, Simon MA, Peabody TD, et al: An orthotopic model of human osteosarcoma growth and spontaneous pulmonary metastasis. Clin Exp Metastasis 22: 319-329, 2005.

15. Livak KJ and Schmittgen TD: Analysis of relative gene expression data using real-time quantitative PCR and the 2(-Delta Delta C(T)) Method. Methods 25: 402-408, 2001.

16. Savage, S.A. and L. Mirabello, Using epidemiology and genomics to understand osteosarcoma etiology. Sarcoma, 2011. 2011: p. 548151 .

17. Ek ET, Dass CR and Choong PF: Commonly used mouse models of osteosarcoma. Crit Rev Oncol Hematol 60: 1-8, 2006.

18. Chen X, Li Y, Xiong K, Aizicovici S, Xie Y, Zhu Q, et al. Cancer gene therapy by direct tumor injections of a nonviral $\mathrm{T} 7$ vector encoding a thymidine kinase gene. Human Gene Ther 1998; 9(5):729-36.

19. Crnalic S, Hakansson I, Boquist L, Lofvenberg R, Brostrom LA. A novel spontaneous metastasis model of human osteosarcoma developed using orthotopic transplantation of intact tumor tissue into tibia of nude mice. Clin Exp Metastasis 1997; 15(2):164-72.

20. Vergote J, Moretti JL, de Vries EG and Garnier-Suillerot A: Comparison of the kinetics of active efflux of $99 \mathrm{mTc}-\mathrm{MIBI}$ in cells with P-glycoprotein-mediated and multidrug-resistance protein-associated multidrug-resistance phenotypes. Eur J Biochem 252: 140-146, 1998

21. Suto R, Abe Y, Nakamura M, Ohnishi Y, Yoshimura M, Lee YH, Imanishi T, Yamazaki H, Kijima H, Tokunaga T, et al: Multidrug resistance mediated by overexpression of P-glycoprotein in human osteosarcoma in vivo. Int J Oncol 12: 287-291, 1998.

22. Chan HS, Grogan TM, Haddad G, DeBoer G and Ling V: P-glycoprotein expression: Critical determinant in the response to osteosarcoma chemotherapy. J Natl Cancer Inst 89: 1706-1715, 1997.

23. Burak Z, Moretti JL, Ersoy O, Sanli U, Kantar M, Tamgac F and Basdemir G: $99 \mathrm{mTc}-\mathrm{MIBI}$ imaging as a predictor of therapy response in osteosarcoma compared with multidrug resistance-associated protein and P-glycoprotein expression. J Nucl Med 44: 1394-1401, 2003.

24. Dutour A, Leclers D, Monteil J, Paraf F, Charissoux JL, Rousseau R and Rigaud M: Non-invasive imaging correlates with histological and molecular characteristics of an osteosarcoma model: Application for early detection and follow-up of MDR phenotype. Anticancer Res 27: 4171-4178, 2007.

25. Beachy PA, Karhadkar SS and Berman DM: Tissue repair and stem cell renewal in carcinogenesis. Nature 432: 324-331, 2004.

26. Ravandi F, Burnett AK, Agura ED and Kantarjian HM: Progress in the treatment of acute myeloid leukemia. Cancer 110: 1900-1910, 2007.

27. Woodward WA and Sulman EP: Cancer stem cells: Markers or biomarkers? Cancer Metastasis Rev 27: 459-470, 2008.

28. Hay DC, Sutherland L, Clark J and Burdon T: Oct-4 knockdown induces similar patterns of endoderm and trophoblast differentiation markers in human and mouse embryonic stem cells. Stem Cells 22: 225-235, 2004.

29. Boyer LA, Lee TI, Cole MF, Johnstone SE, Levine SS, Zucker JP, Guenther MG, Kumar RM, Murray HL, Jenner RG, et al: Core transcriptional regulatory circuitry in human embryonic stem cells. Cell 122: 947-956, 2005.

30. Wei H, Zhao MQ, Dong W, Yang Y and Li JS: Expression of c-kit protein and mutational status of the c-kit gene in osteosarcoma and their clinicopathological significance. J Int Med Res 36: 1008-1014, 2008 